\title{
Nitrogen uptake in temperate heath vegetation and soil microbes is influenced by elevated temperature, $\mathrm{CO} 2$ and drought
}

\author{
Andresen, L.C.; Michelsen, Anders; Johansson, Sven; Beier, Claus; Ambus, Per
}

Publication date:

2009

Link back to DTU Orbit

Citation (APA):

Andresen, L. C., Michelsen, A., Johansson, S., Beier, C., \& Ambus, P. (2009). Nitrogen uptake in temperate heath vegetation and soil microbes is influenced by elevated temperature, CO2 and drought. Poster session presented at BIOGEOMON 2009, University of Helsinki (FI), 29 Jun - 3 Jul.

\section{General rights}

Copyright and moral rights for the publications made accessible in the public portal are retained by the authors and/or other copyright owners and it is a condition of accessing publications that users recognise and abide by the legal requirements associated with these rights.

- Users may download and print one copy of any publication from the public portal for the purpose of private study or research.

- You may not further distribute the material or use it for any profit-making activity or commercial gain

- You may freely distribute the URL identifying the publication in the public portal 


\section{Nitrogen uptake in temperate heath vegetation and soil microbes is influenced by elevated temperature, $\mathrm{CO}_{2}$ and drought}

$\underline{1}$ Louise C. Andresen, ${ }^{1}$ Anders Michelsen, ${ }^{1}$ Sven Jonasson, ${ }^{2}$ Claus Beier, ${ }^{2}$ Per Ambus

1 University of Copenhagen, Denmark; and

2 Risø National Laboratory for Sustainable Energy, Technical University of Denmark, Denmark

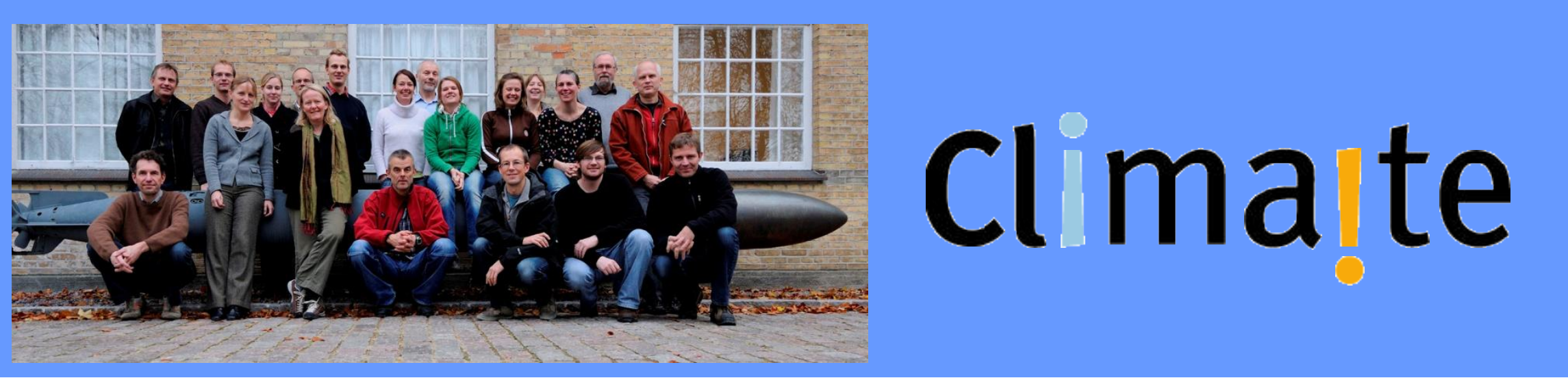




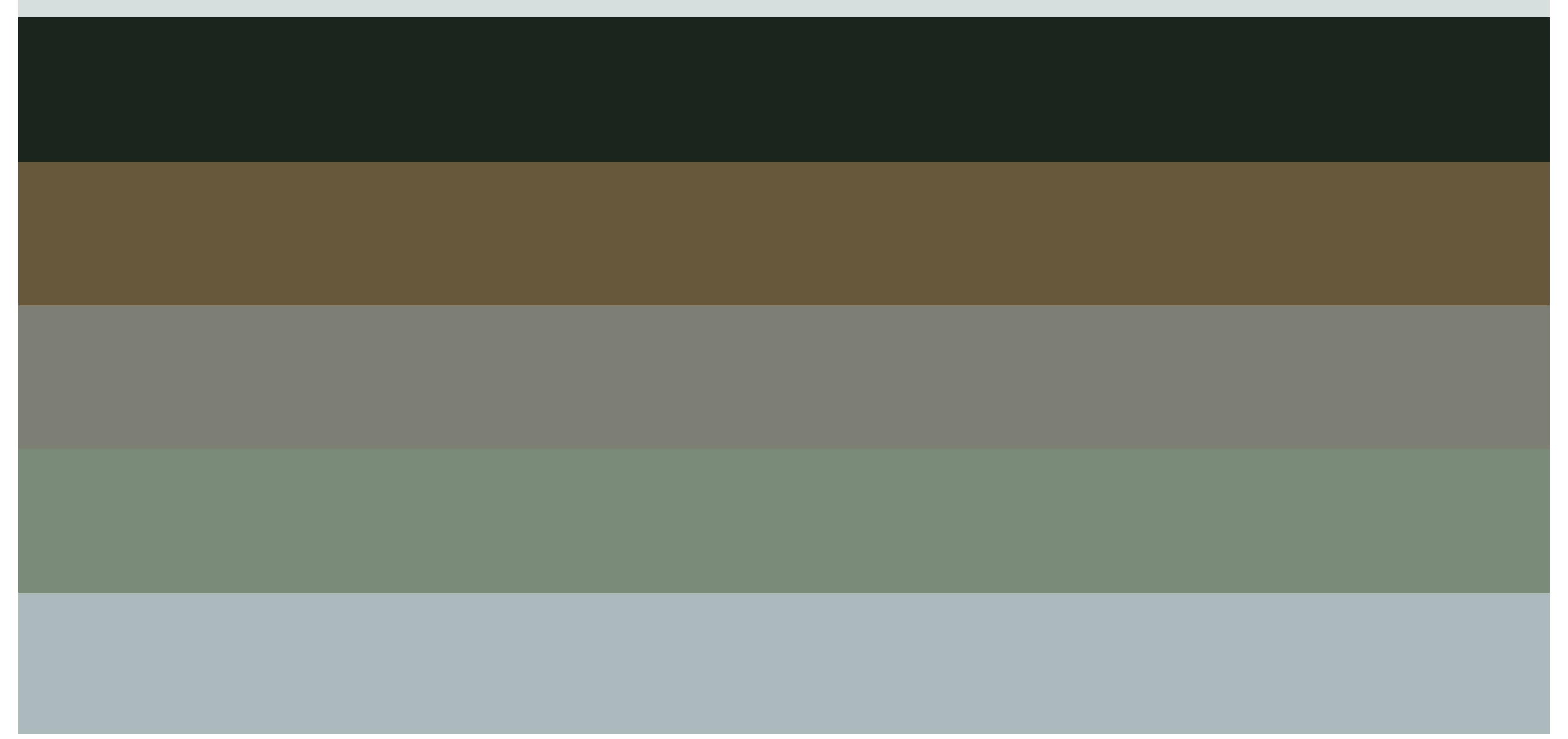




\section{Micobes: no effect from fertilization}

Roots increase in biomass with $\mathrm{N}$ and NNP

\section{Applied Soil Ecology}

(2009); vol $42279-287$

Nielsen, Andresen,

Michelsen, Schmidt and

Kongstad

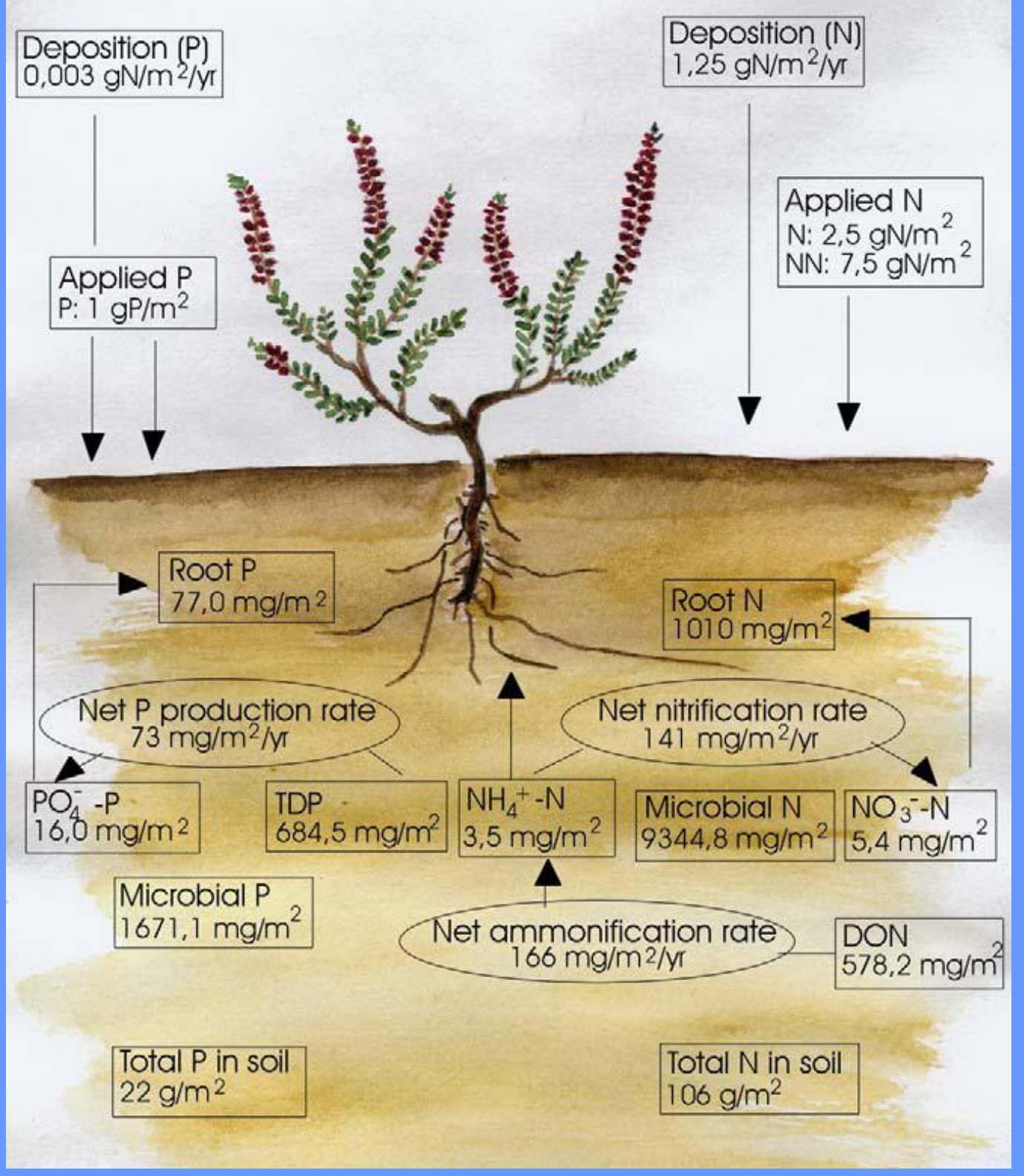




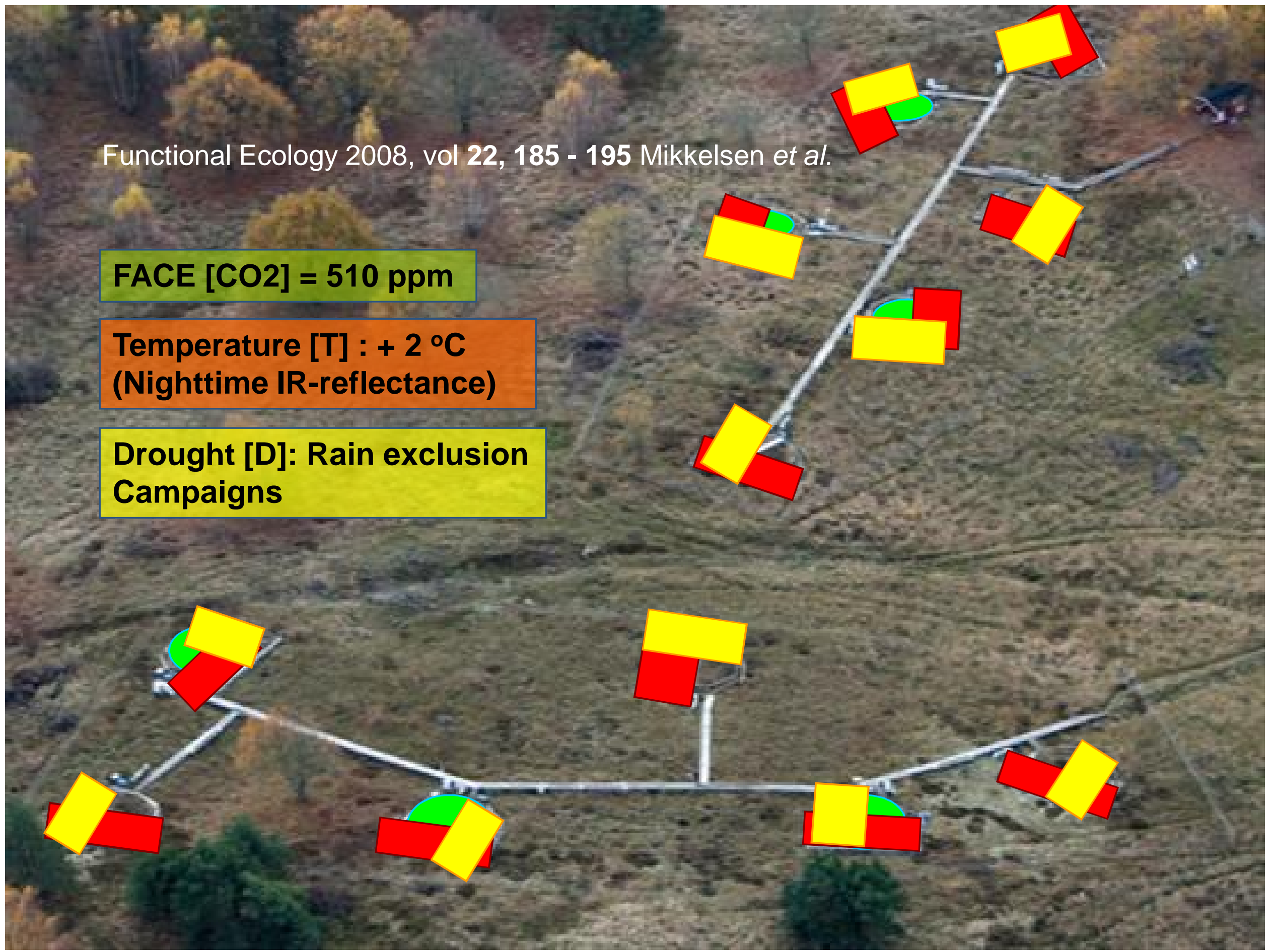




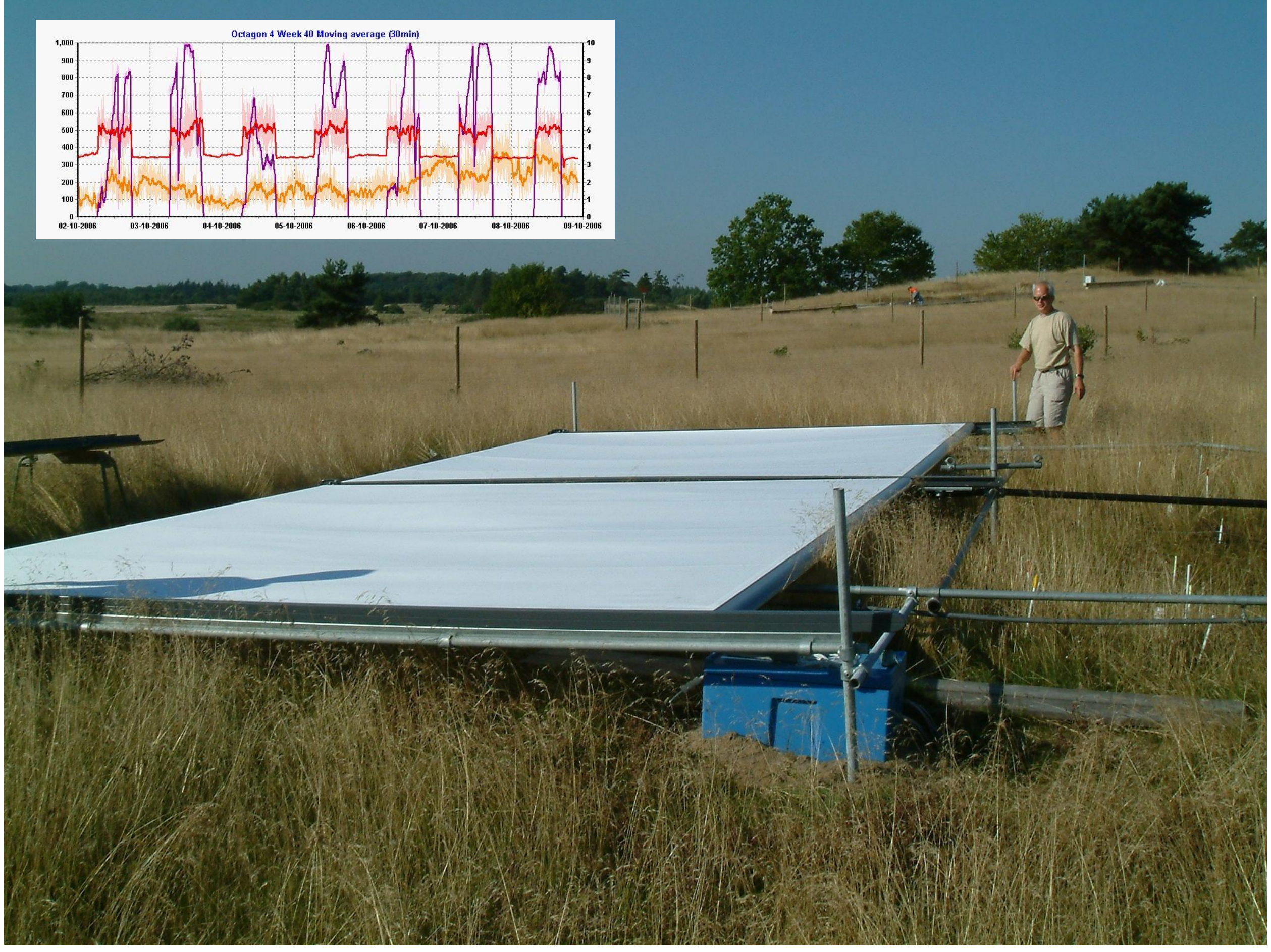




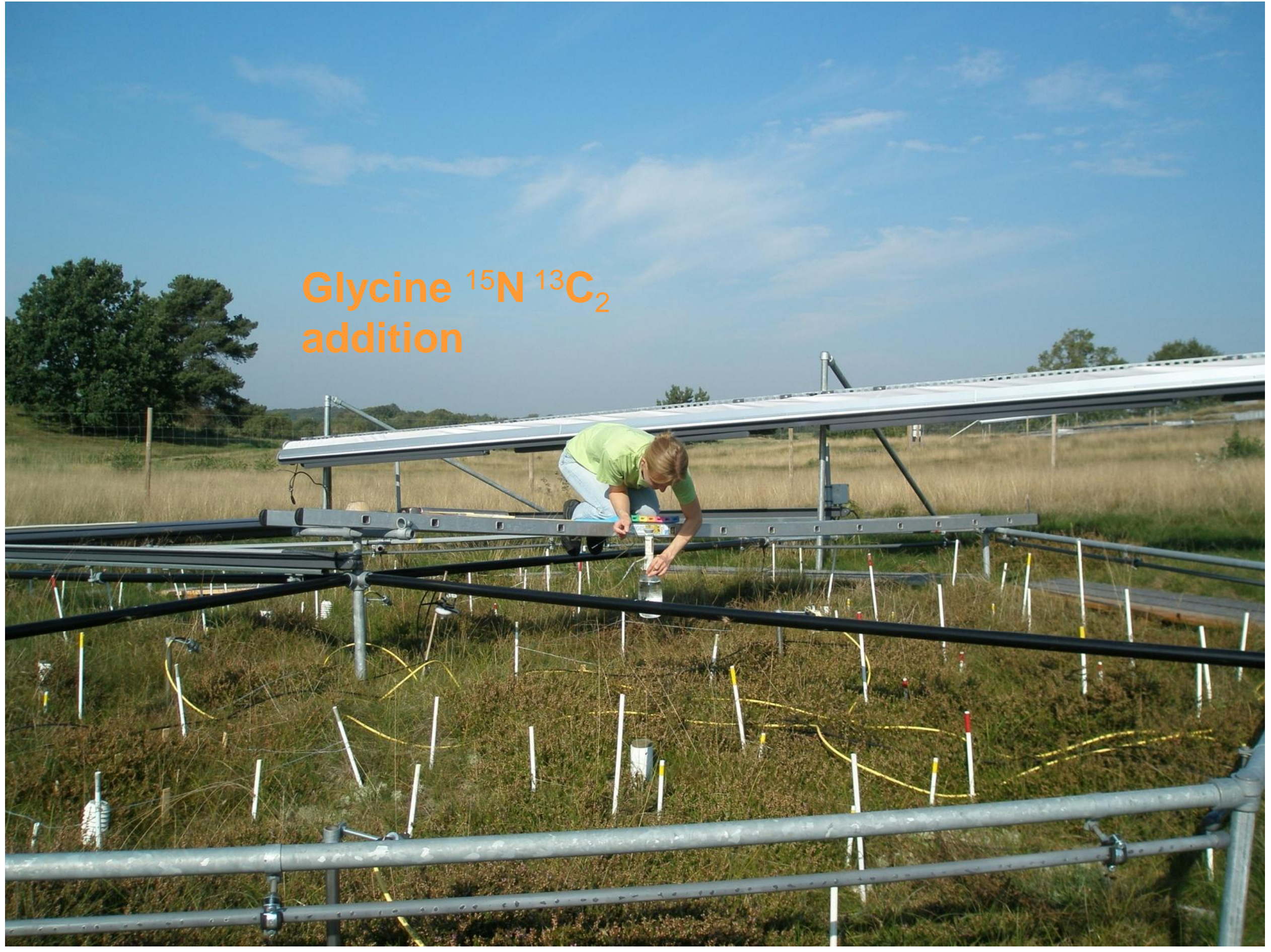




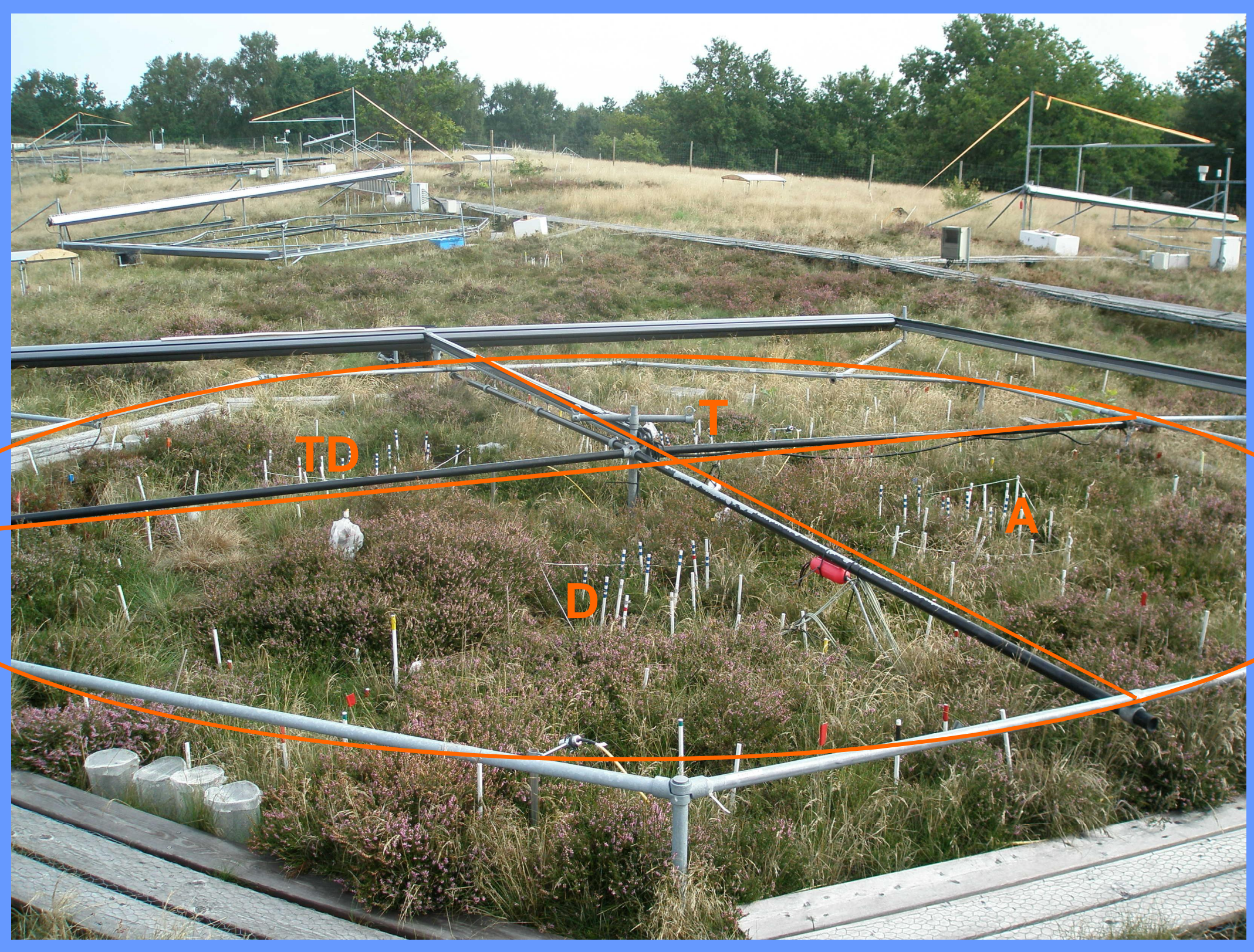




\section{Immediate}

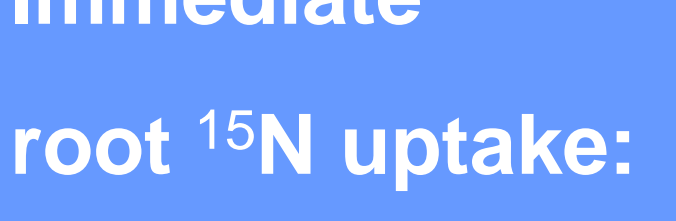

$\mathbf{T} \uparrow$

$\mathrm{CO} 2 \uparrow$

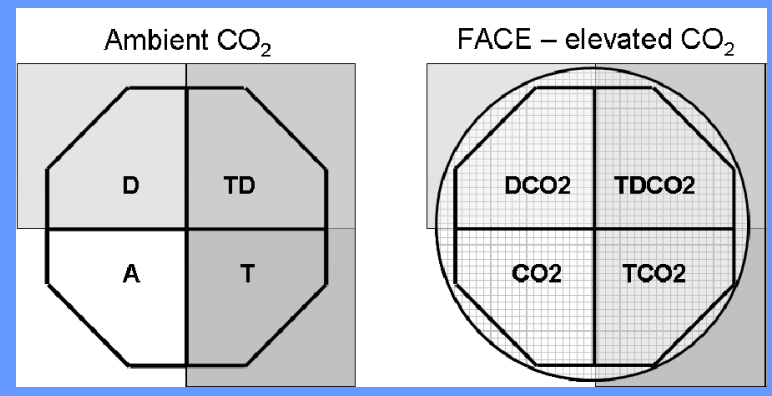

Submitted to Acta Oecologica (2009)

Andresen, Michelsen, Jonasson, Ambus,

Beier

\section{a}

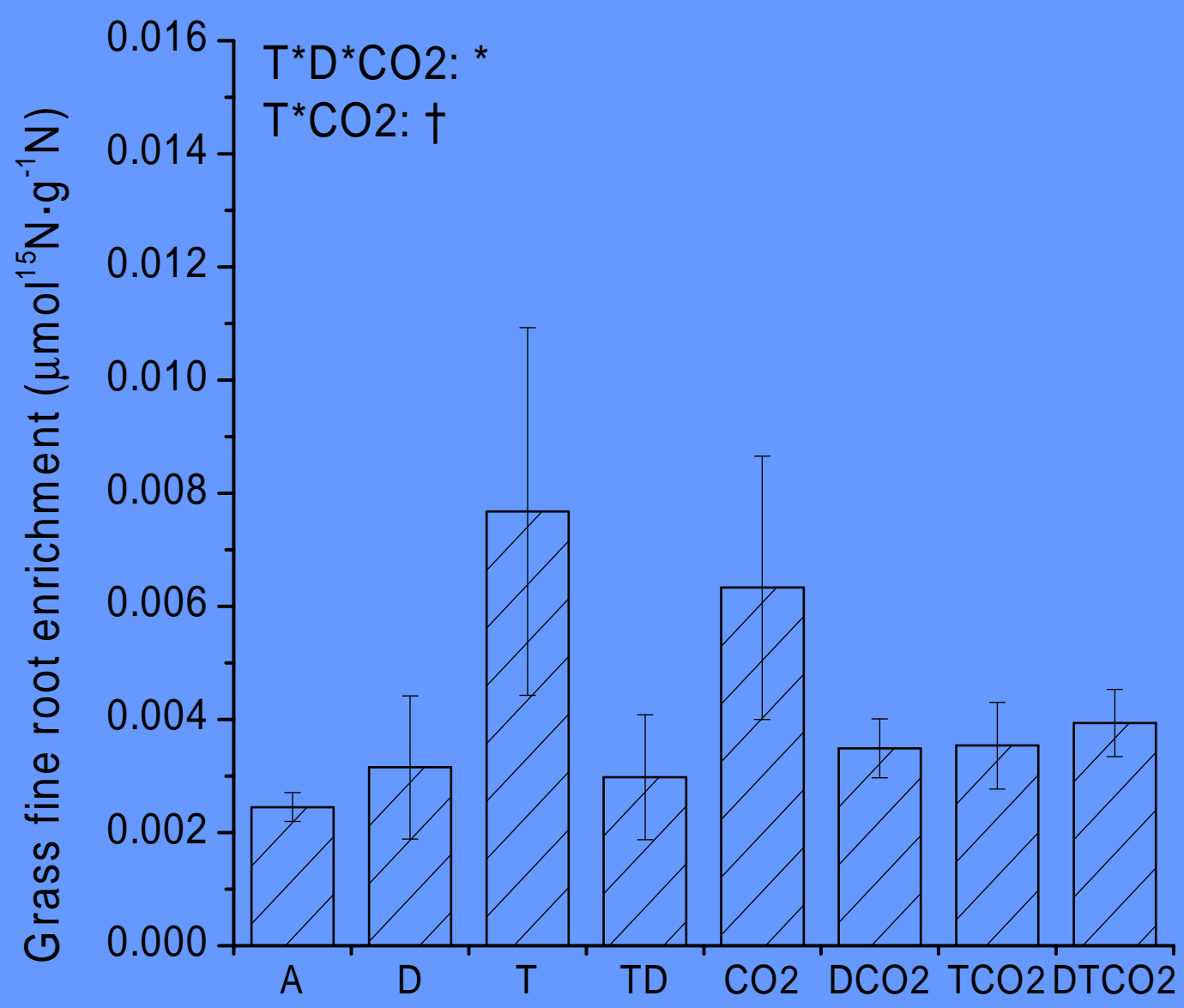




\section{BIOGEOMON 2009}

Ammonium

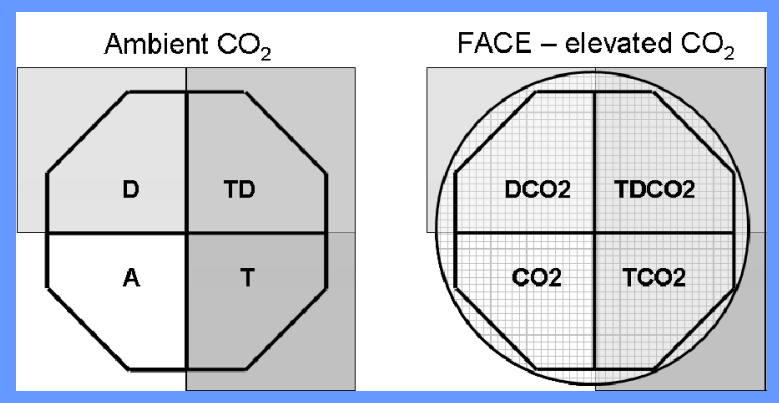

To be submitted, Andresen et al.

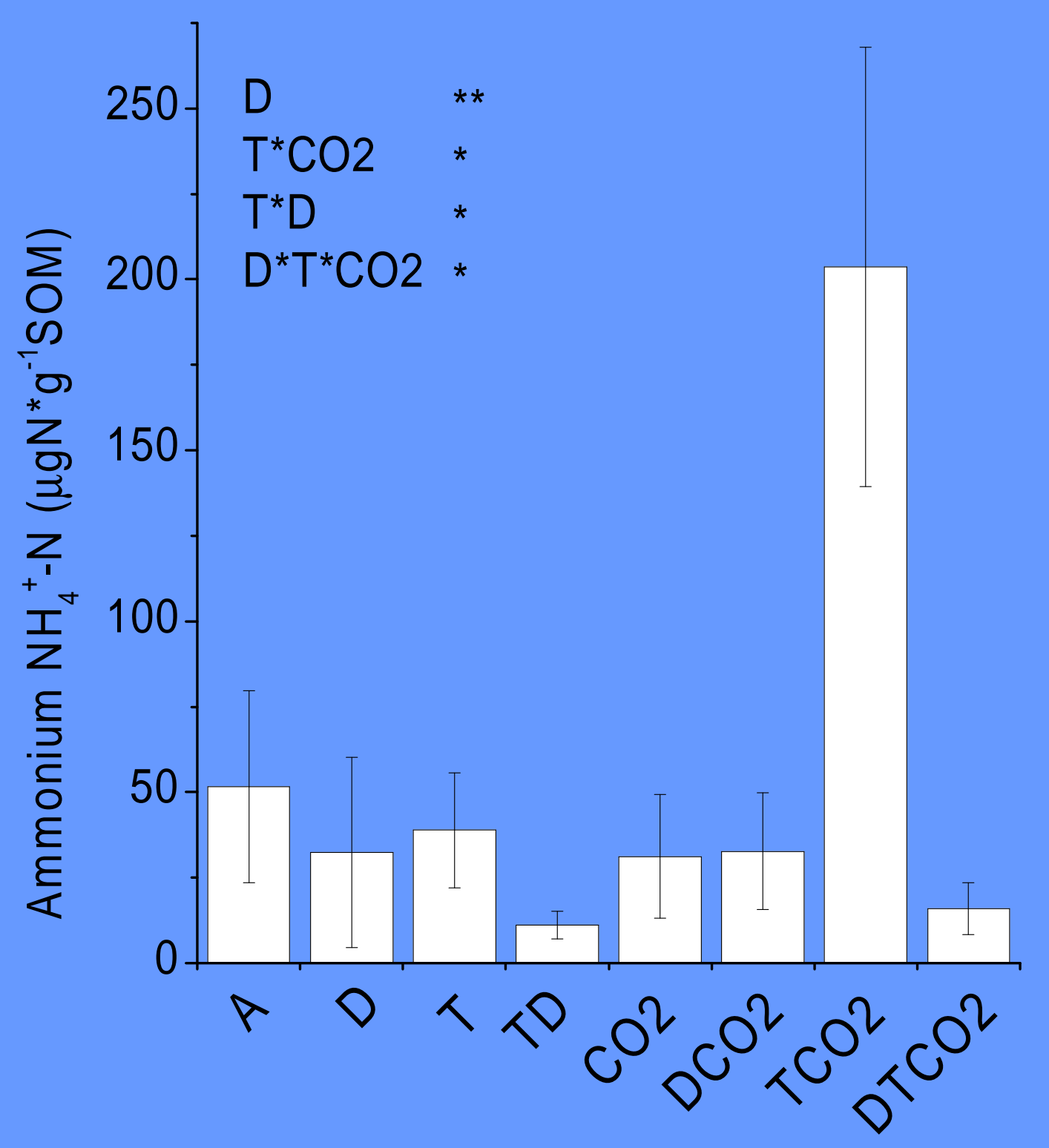




\section{BIOGEOMON 2009}

\section{DON:}

TCO2 $\downarrow$

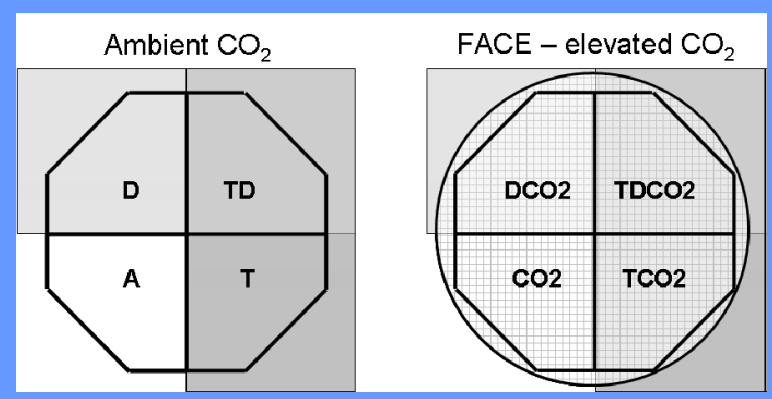

To be submitted, Andresen et al.

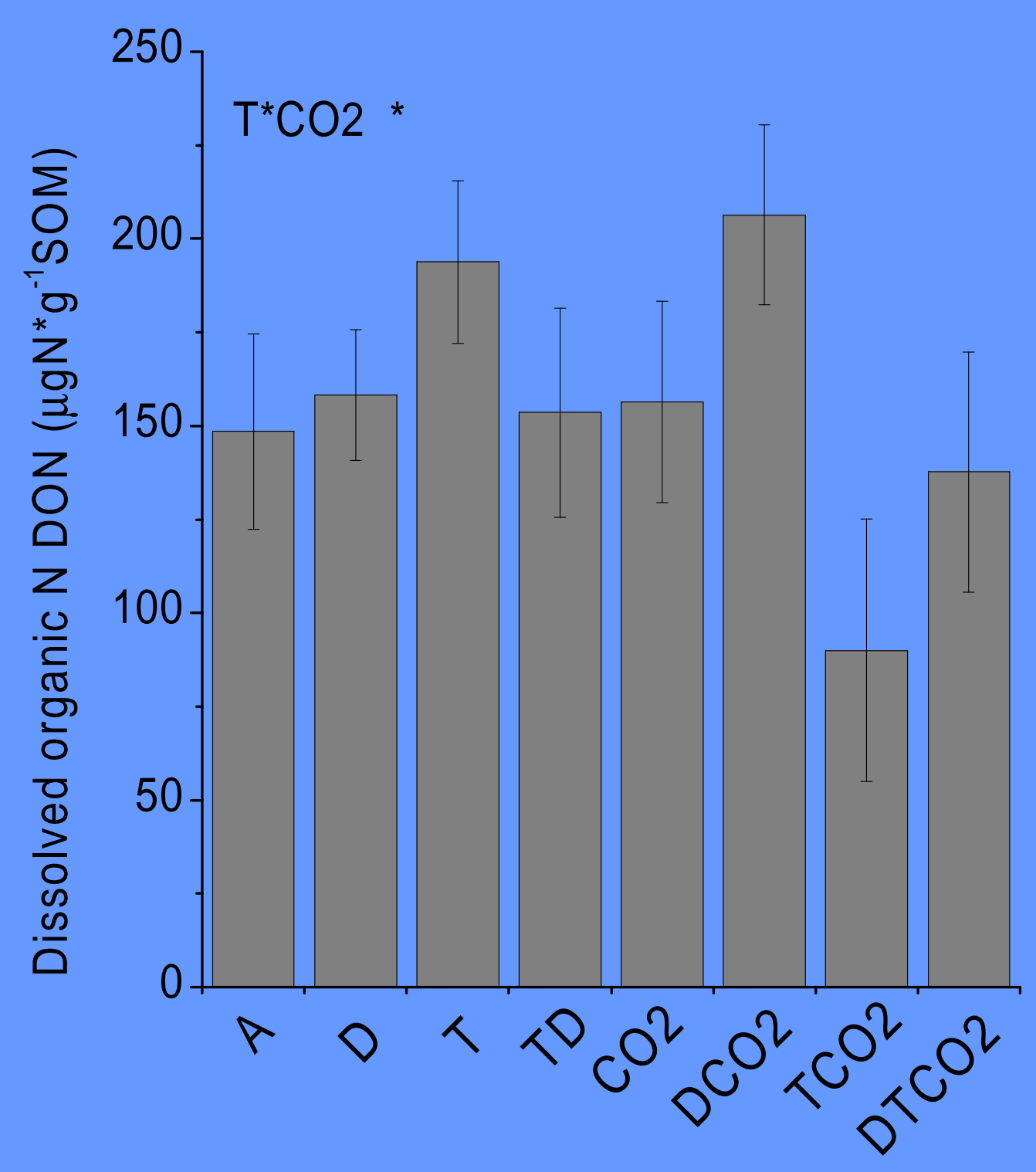




\section{BIOGEOMON 2009}

Microbial

\section{carbon:}

$\mathbf{T} \uparrow$

$\mathrm{CO} 2 \uparrow$

TCO2 --

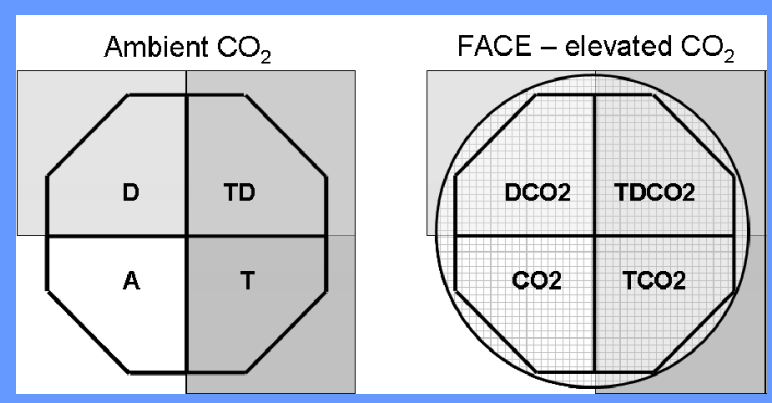

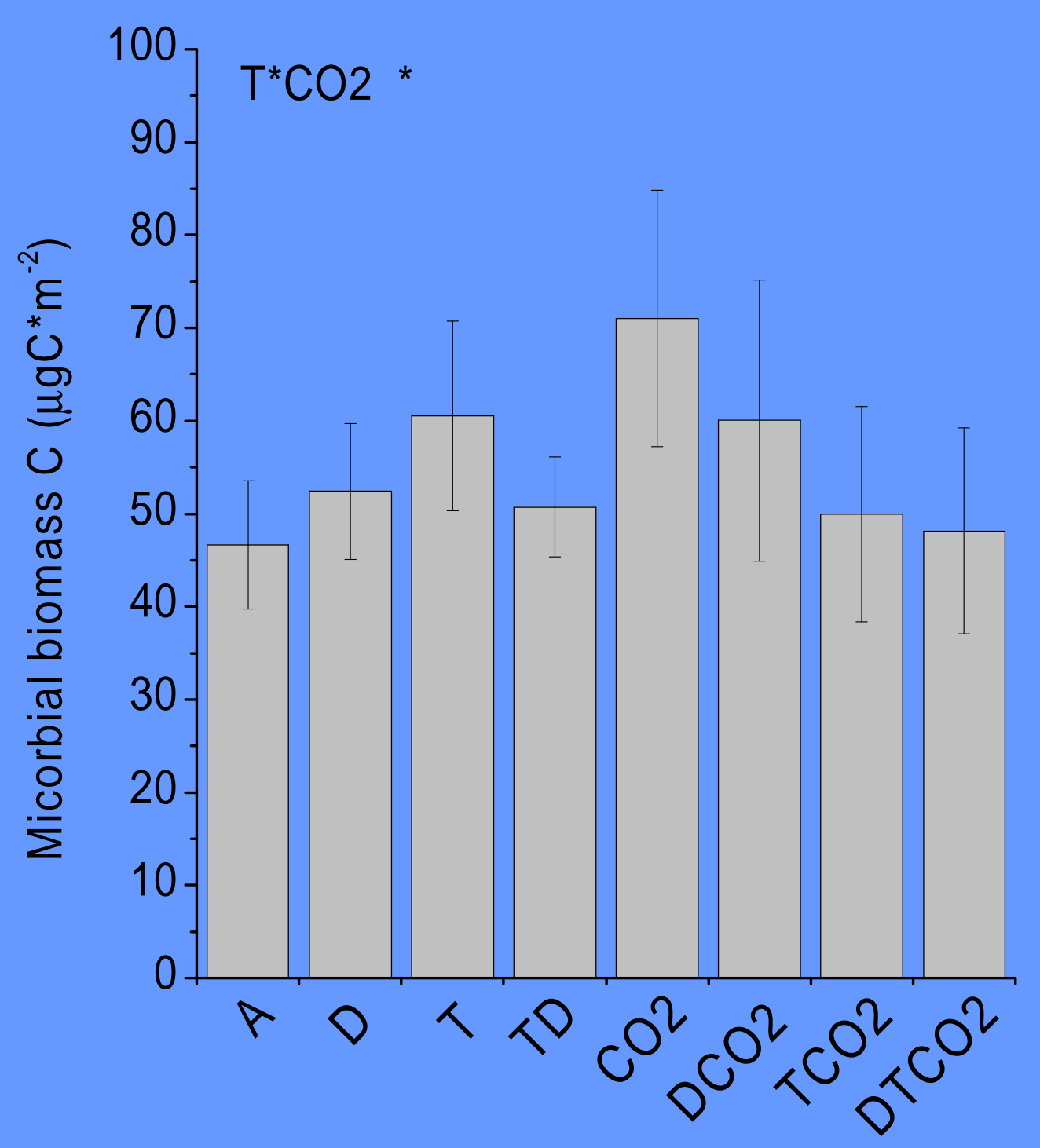

To be submitted, Andresen et al. 


\section{BI OGEOMON 2009}

Heather

flower $\mathrm{N} \%$ :

$\mathrm{CO} 2 \downarrow$

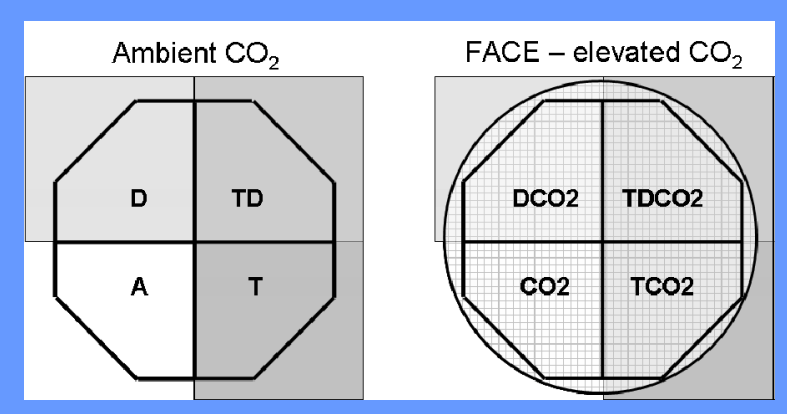

To be submitted, Andresen et al.

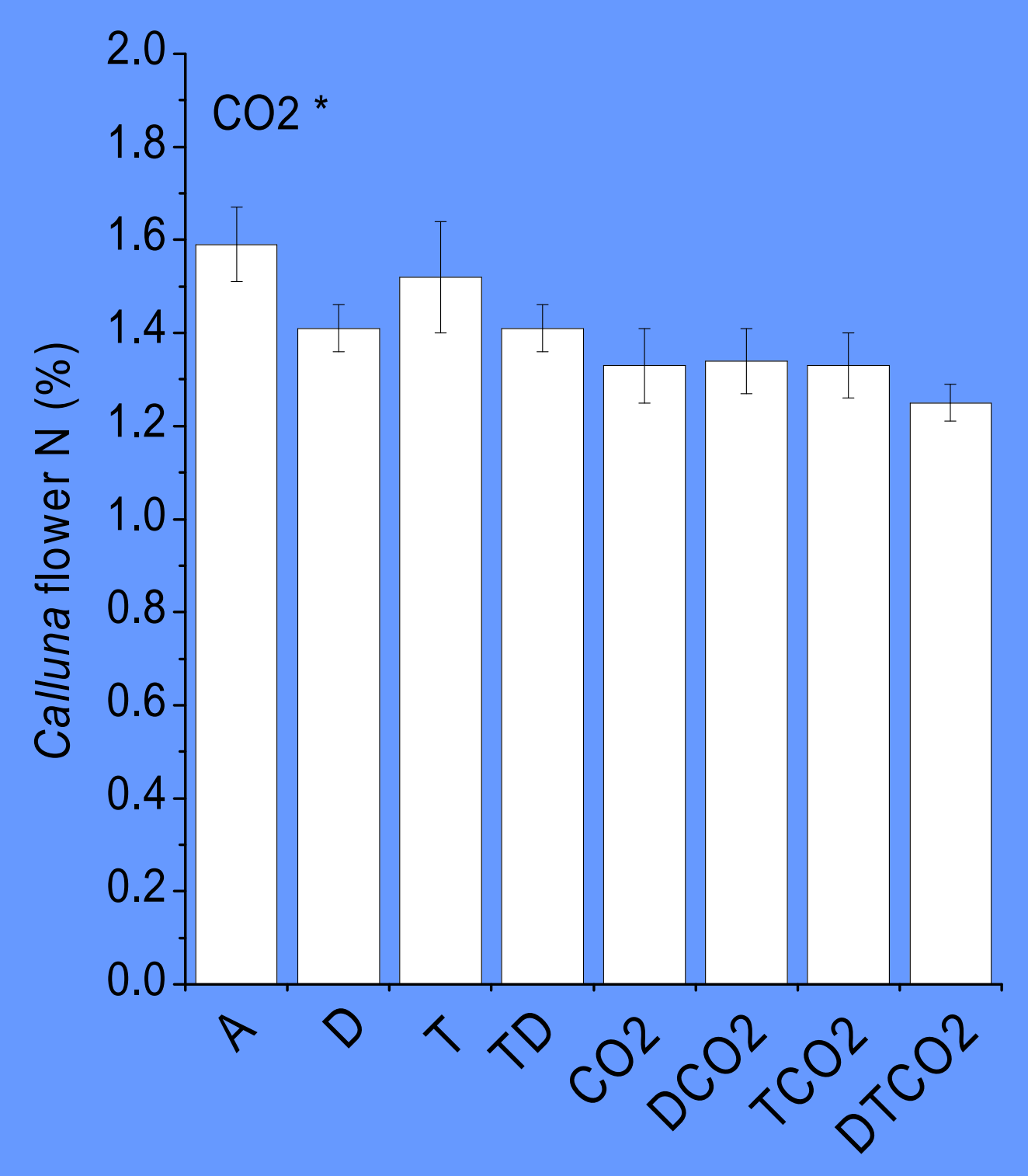




\section{BIOGEOMON 2009}

\section{Heather}

N pool:

D $\uparrow$

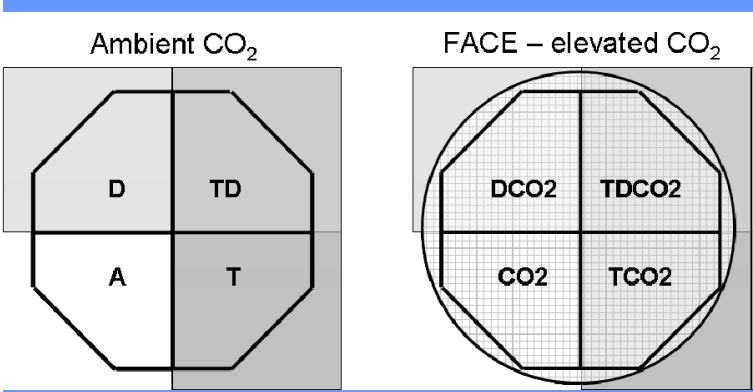

To be submitted, Andresen et al.

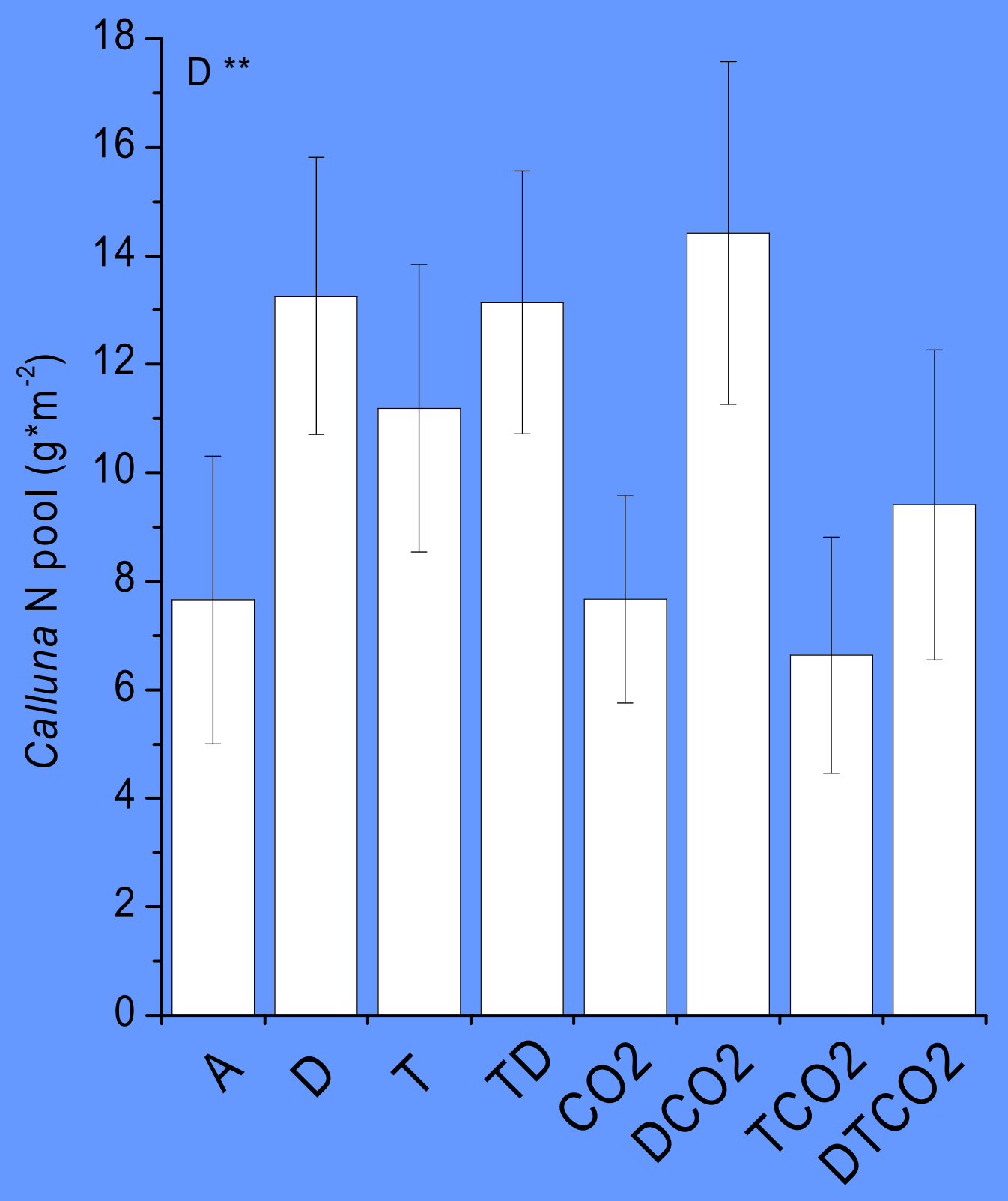




\section{Heather}

${ }^{15} \mathrm{~N}$ recovery:

$\mathbf{D} \uparrow$

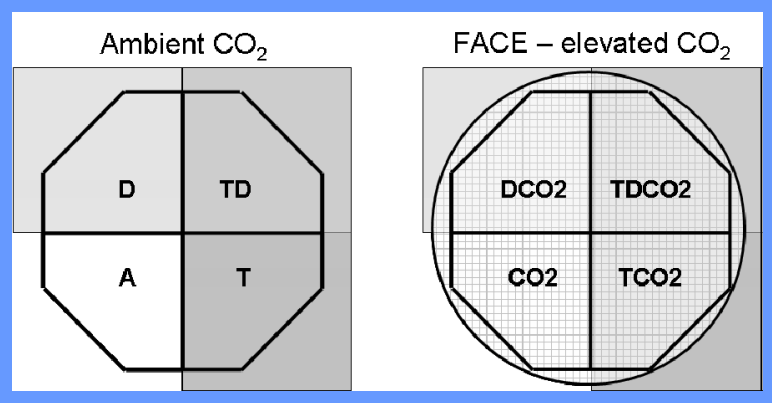

To be submitted, Andresen et al.

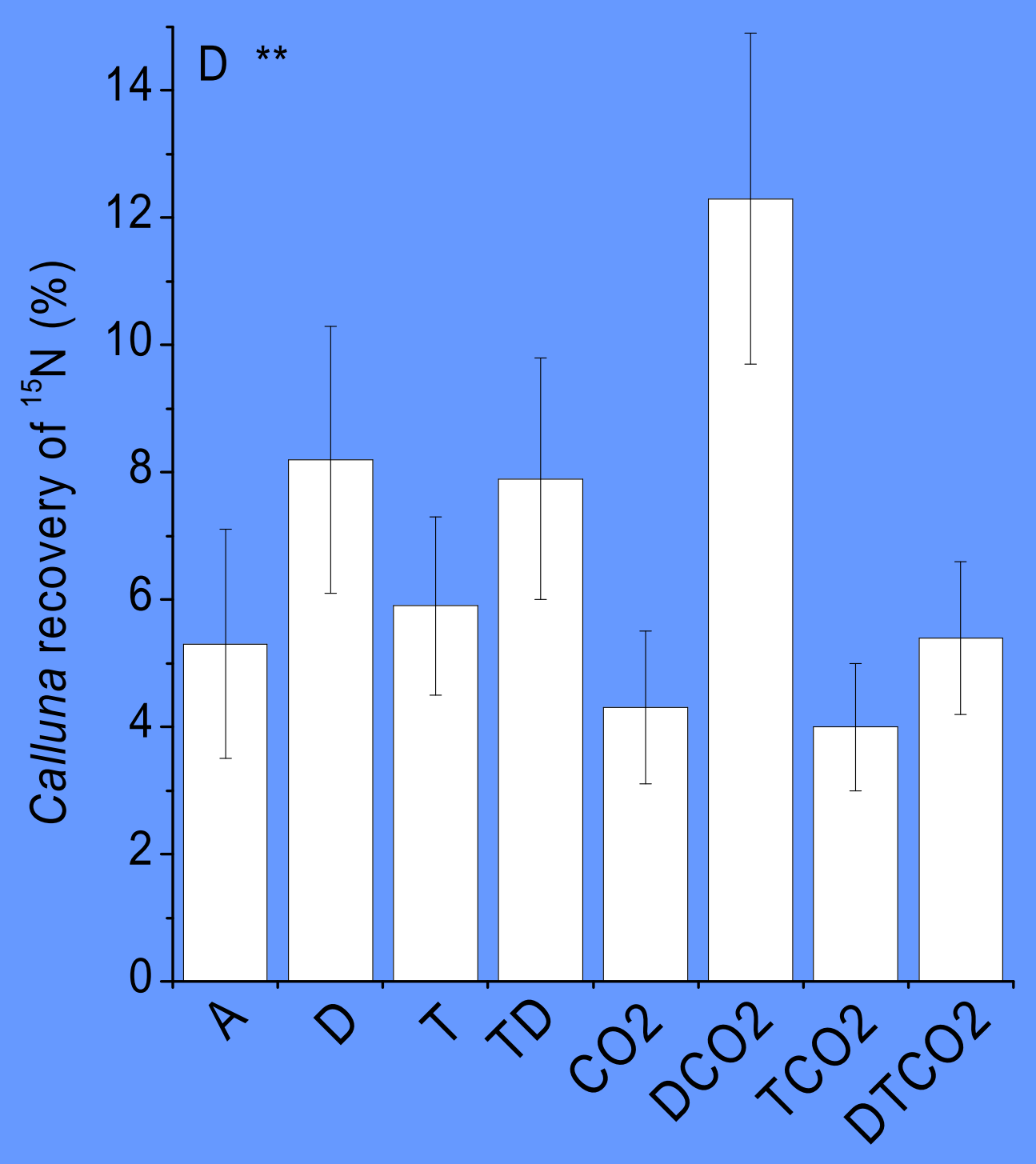




\section{Nitrification}

\section{Nitrification $\left(\Delta \mathrm{NO}_{3}-\mathrm{N}\right)$ rates}

rate:

$\mathbf{D} \downarrow$

\section{ALSO litter} decomposition:

$\mathbf{D} \downarrow$
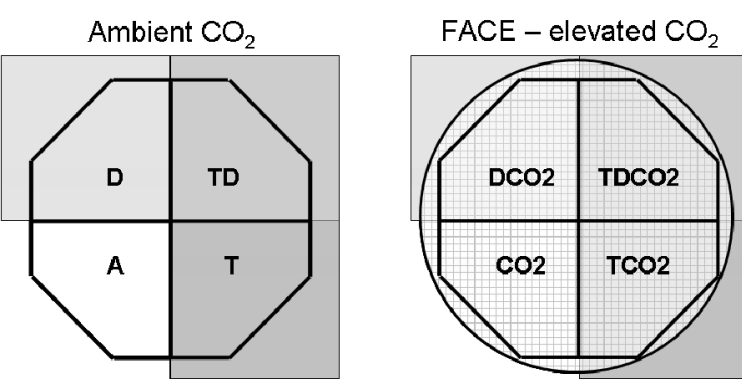

$0.6] \mathrm{D}: * *$

$-0.2$

D

T

TD

CO2 DCO2 TCO2 DTCO2

$1 / 2$ year incubated Deschampsia soil with no plants

Submitted to Plant and Soil (2009)

Andresen, Michelsen, Jonasson,

Mikkelsen, Schmidt, Ambus, Beier 


\section{BIOGEOMON 2009}

Microbial ${ }^{15} \mathbf{N}$ recovery:

$\mathrm{T} \uparrow$

$\mathrm{CO} 2 \uparrow$

TCO2 --

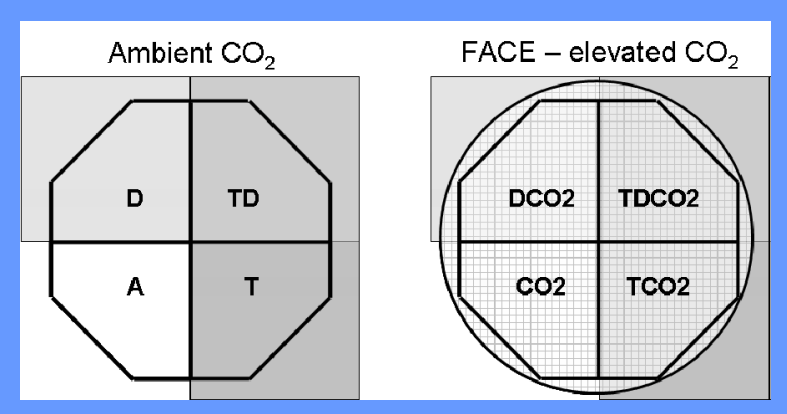

To be submitted, Andresen et al.

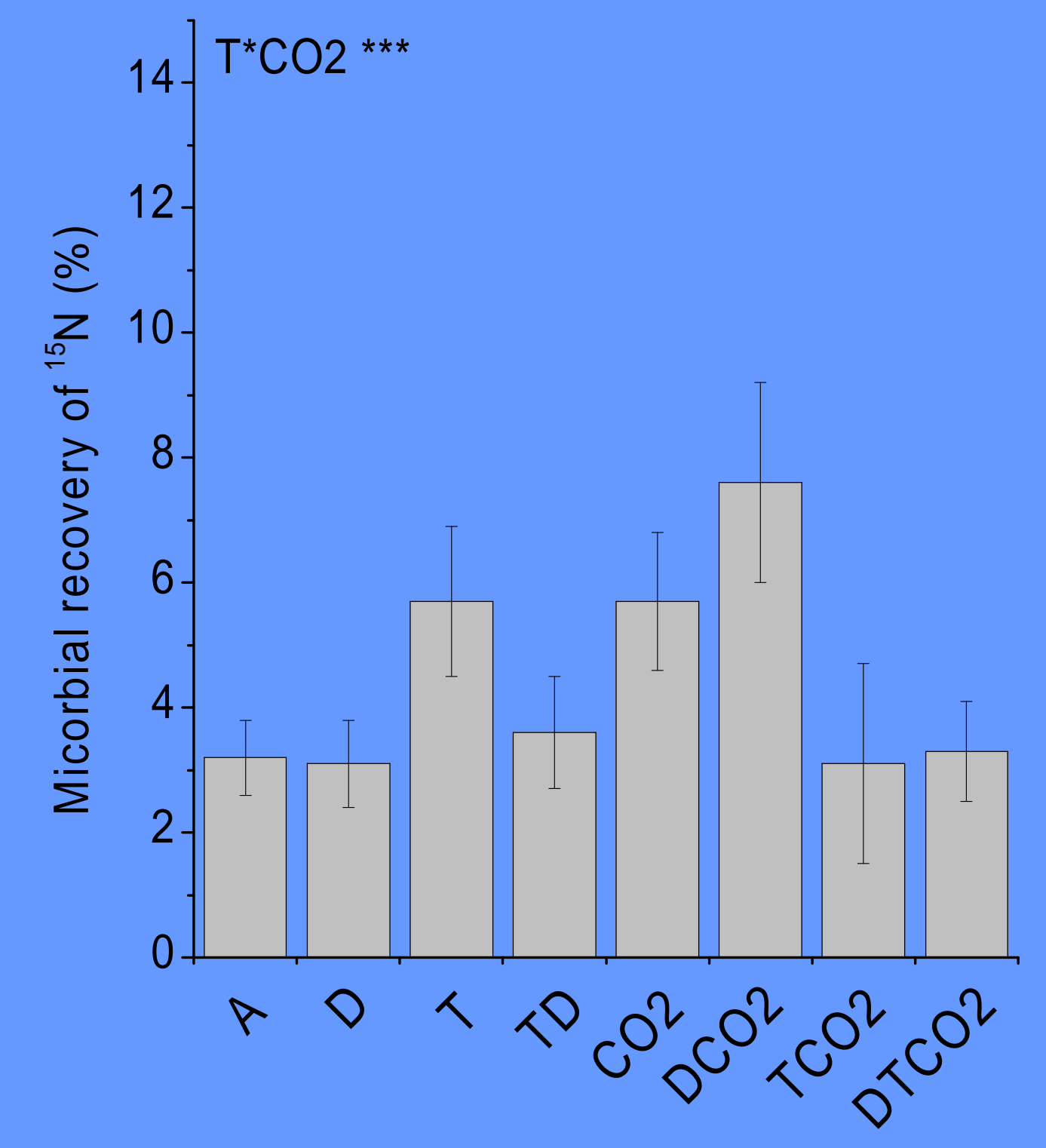


TWO years of climate change treatments:

- Combined warming and elevated $\mathrm{CO}_{2}$ kicks up mineralization of DON into ammonium

- Microbial biomass $\mathrm{C}$ and ${ }^{15} \mathrm{~N}$ tracer recovery higher in warmed and elevated $\mathrm{CO}_{2}$ plots (not in $\mathrm{TCO}_{2}$ )

- $\mathrm{CO}_{2}$ dilutes nitrogen in Heather flowers (and fine roots)

- Drought increases Heather N pool, biomass and tracer recovery 
Papers from the field site:

Experimental design: Mikkelsen et al.

Functional Ecology 2008, vol 22, 185 - 195.

$\mathrm{N}$ and $\mathrm{P}$ application: Nielsen et al. Applied

Soil Ecology 2009, vol 42, $279-287$.

Louise C. Andresen:

and 


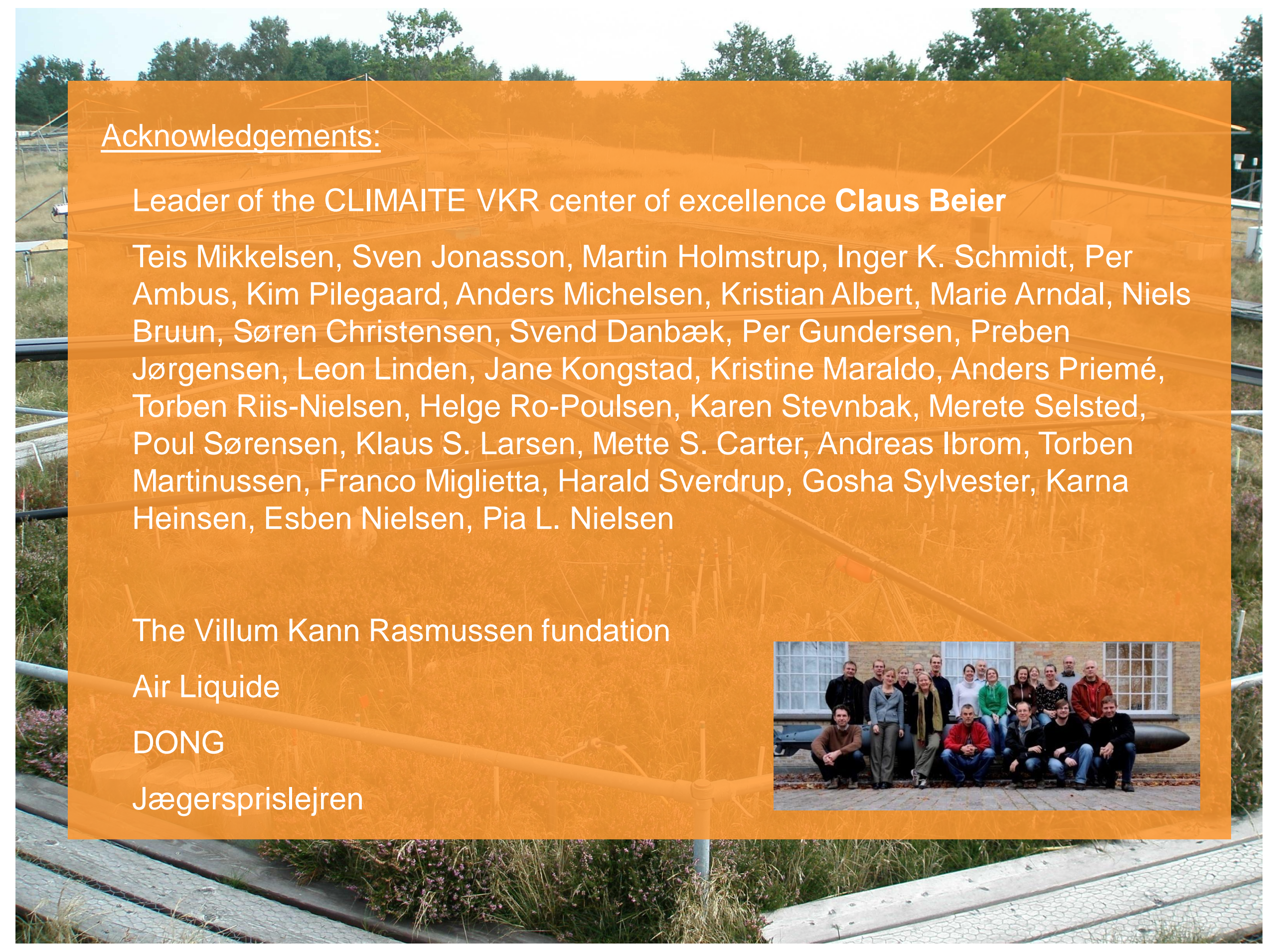

